\title{
Real Time Monitoring and Controlling of the Hazardous Places by using Virtual Agent
}

\author{
Navneet Tiwari, Member, IACSIT, Jatan Saraf
}

\begin{abstract}
Take safety precautions, be alert always, and keep your eyes open etc. is some of the most common signboards seen in industries. True, most of the precautions are followed as well, but it needs to be understood that this may not be enough. Some of the risk is unavoidable in the daily life. Further when we are talking about life risks, we can not neglect the threat of terrorist activity, bombing cases and biological weapon attacks that are becoming quite regrettably very common these days. In Indian Scenario the problem get more worsen out. This all such events, where the lives of thousands of people depends on just the action and monitoring of few courageous people. Though, I am not questioning their guts, but when the lives of so many individuals are concerned, the liberty of error is not even marginal. Because, the human resource is one of the most important resource of each country. India is the one of the richest country on human resource domain.

This paper deals with the designing of, "Area surveillance rover over the wireless channel ", which is considered as a virtual agent. We all know that there are a number of machines in working conditions right now that are being used for the hazardous job. But all of them are extremely task specific. Now what if, we have a platform that can be made to monitor all such activities which up till now were done by the humans. Thus, making it safe and affordable for exploration of various exploring places like mine, excavation places etc. In this paper, I am representing a working model of this feasible machine. It is not the ultimate model; but in simple words, I am looking for the endless opportunity.
\end{abstract}

Index Terms - CODEC, CMOS Camera, Differential Manchester Encoding, RF Module, Wireless Channel.

\section{INTRODUCTION}

The modern world of industries with its growing requirement of raw materials and ores is increasingly demanding more and more excavations projects to undergo. It is an evident fact that exploration phase of every such projects engages serious life risk of people involved in it. But it is a risk and we are constrained to do so.

But surely, we can not expect a person to show his full unaffected ness to his work; when he or she knows it pretty well that, his life is hanging with just a rope or his only source of contact between his life and death is walky - talky. The continuous, interactive and integrative research

*Manuscript Submitted on 20 June 2009.

*Manuscript Accepted for Publication on 29 July 2009.

A. Author is with the Medi-Caps Institute of Technology and Management, Indore 452009 ,India. [Corresponding author phone: 91-98260 79288; e-mail: navneet1231@gmail.com].

B. Author is with the Medi-Caps Institute of Technology and Management, Indore 452009,India [e-mail:sarafjatan@yahoo.co.in] effort always improves performance and provides a new possibility. A sensible, robust risk measurement technology ensures risks are transparent. Risk transparency ensures that high risk derivative instruments cannot be packaged as safe investments. The implementation of, "Area Surveillance Rover over wireless channel" can be made to monitor all such activities which up till now where done by human. There are countless advantages of this system.A few of them are demonstrated in the following points:

[A]. The one of the most important advantage is that, it reduces the risk of the human life. Thus, making it affordable for exploration of the areas where until now they where not used because of the financial considerations.

[B]. From a single control unit, we can easily monitor the machines in Industry places. Thus, repairing and the maintenance of the machine will be much cheaper, afford -able and will not demand separate special people for monitoring over them .If we can keep it simple then we need not people trained to explore them .A simple manual will do the job and the machine may become household equipment.

[C]. It will required less amount of time and reduces the life danger from severe critical condition.

Above are just a few advantage that, we have pointed out. The opportunities are endless and count less. [8] And, this was the source of inspiration of this paper. A machine that was so general that, we can make it for the exploration of various hazardous places. For example, if we need to survey a newly discovered mine, we can simply attach a camera or a temperature or a climate sensor over our plat form and send it into the mine. Now, after the job is over, a terrorist threat comes up, now we can be able to send it over the threat area .By controlling it from, the main frame unit we can easily find out the hiding places of terrorist. The task, which until now demanded the human life risk become risk free, safe and reliable. Isn't it effective or not?

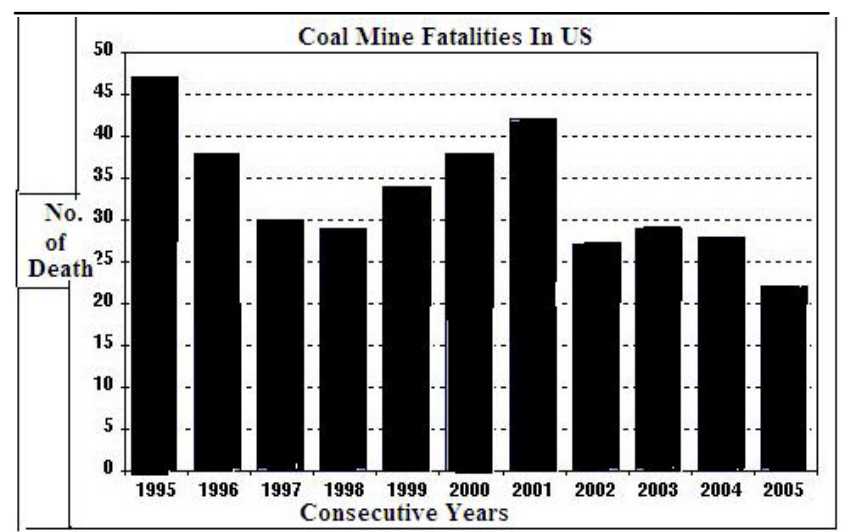

Fig. 1 Demonstration of the coal mine fatalities in the United States of America during the period of 1995-2000. 
Thus, the aim of this paper is to provide a low risk-involving path for these jobs. Device that can be put to use in any such condition excluding with the few exception, which ask for a life risk. A device that can be taken to any terrain and can be made to monitor any kind of job as per requirement [2] .It can be scaled down or scaled up according to the burden of job. The level of accuracy in its performance is also coarse. For the precision work again the accuracy can be increased.

\section{Design CONSIDERATION OF MACHINE}

The "Area Surveillance Rover", is the representation of the pc controlled wireless mobile platform . The platform is four wheeled with two dc motors. Each motor provided power to two wheels, thus, giving a complete four wheel drive mechanism. A Graphical User Interface [GUI] Window is used out for the controlling if the rover. That means, we are using software which were designed on the Visual Basic 6.0. It consist a graphical user interface [GUI]. [4]

\section{A. Being Wireless}

The communication channel is one of the important design criteria. Generally, there are the two propagation channel path are available, either wired or wireless. [1] This paper deals with the wireless implementation of the area surveillance rover. The IEEE $802.11 \mathrm{~b}$ is one of the widely acceptable standards for the wireless communication in the radio frequency range. The wireless section consists out 433 MHz ASK RF modules. [3] This wireless module consists of a transmitter and receiver section, which were operates at the carrier frequency of $433.12 \mathrm{MHz}$ 's. The differential Manchester encoding technique is used for the wireless encoding of data. The Differential Manchester encoding (also known as CDP; Conditioned Diphase encoding) is a method of encoding data in which data and clock signals are combined to form a single self-synchronizing data stream.[4] It is a differential encoding, using the presence or absence of transitions to indicate logical value. This gives it several advantages over vanilla Manchester encoding.

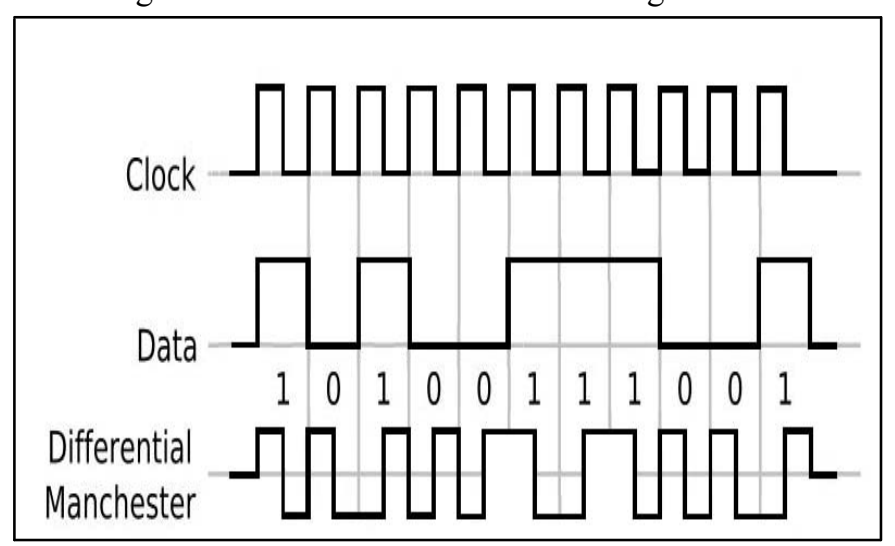

Fig. 2 Representation of the Differential Manchester Encoding Technique.

\section{B. Mobile Platform}

The platform is a four- wheel drive systems consisting of two DC motors, each providing power to one side of the drive. Each motors feeds the torque to the wheels on its side. The motors are fed through the power supply, in steps so as to keep the acceleration within control .In absence of it, the motor may get out of control because of the speed gaining. [2] Thus, the motors are provided power only for that duration in which it is not able to accelerate beyond the control of the driver. The control signal to give power to the motor is given through the computer. The control signal is generated by the developed graphical user interface window and then the power is provide for duration of 200 milliseconds. For again making the motor move, the control signal needs to be given again.

\section{Rotary System}

In a rotary system the purpose of making available a stepper motor is to enable the user with a rotator gearing system .On which the suitable device can be mounted and used according to utility of the user has thought of. The motor rotates in steps for each command taking one step of predefined angles on each push of the button.

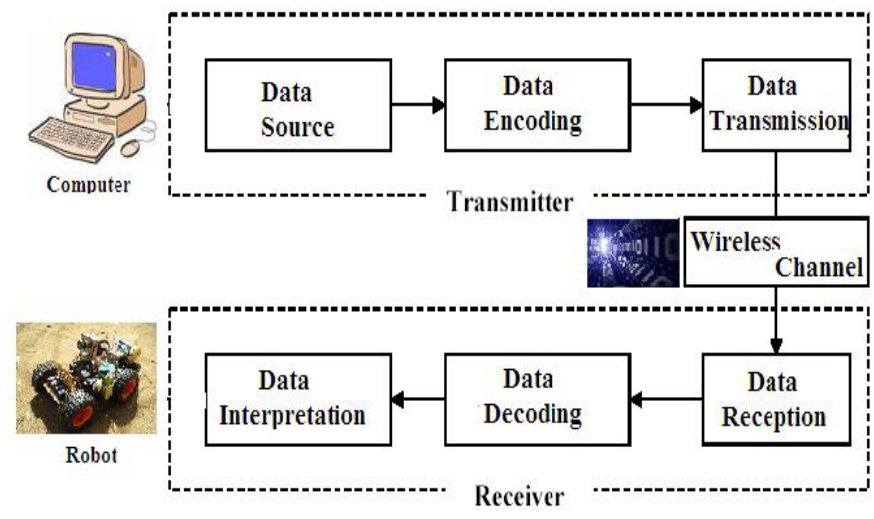

Fig. 3 Representation of the Wireless communication Channel between the transmitter and the receiver unit.

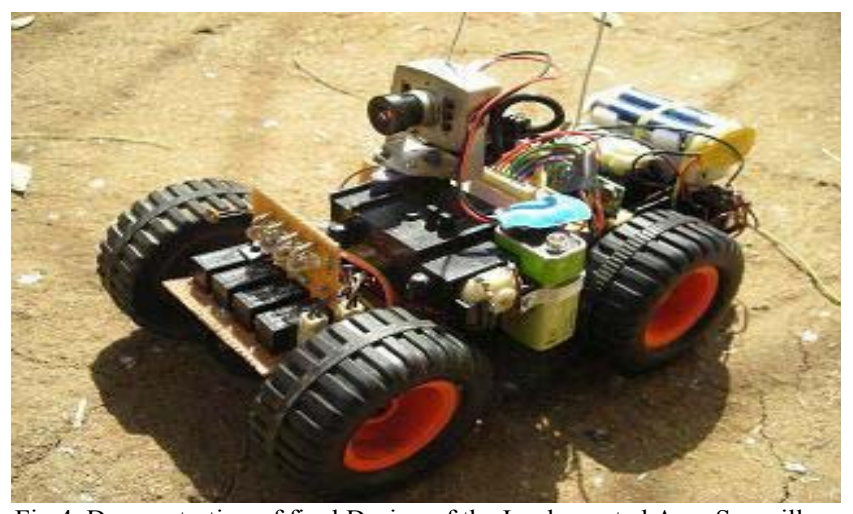

Fig.4 Demonstration of final Design of the Implemented Area Surveillance Rover.

\section{Proposed Design ARChitecture}

In this section, the design details of the transmitter and the receiver section of the Area Surveillance rover is discussed.The whole system follows the following flow chart. 


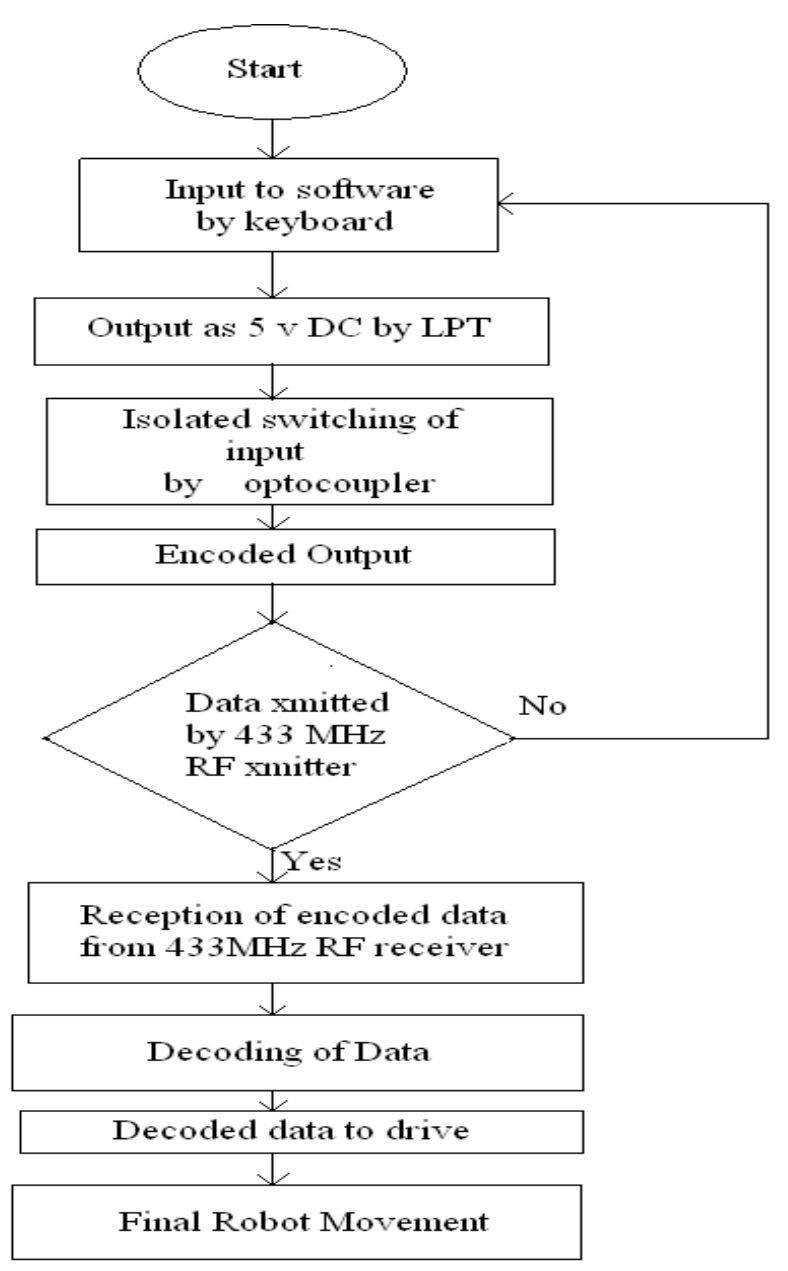

Fig. 5 Flow Chart representing the working of Area Surveillance Rover over Wireless channels.

\section{A. Transmitter Section}

The Parallel Port is generally used for the interfacing of the transmitter section with the computer unit. Parallel port will allow the input of up to 9 bits or the output of 12 bits at any one given time, thus requiring minimal external circuitry to implement many simpler tasks. The port is composed of 4 control lines, 5 status lines and 8 data lines. It's found commonly on the back of your PC as a DB-Type-25 Pin female connector. In the transmitter circuit, the DB 25 connector is interfaced with the MCT2E series opto-coupler. The opto-coupler is used to provide the proper isolation between the ST12E encoder and the parallel port. [1] The input control signal is generated by the graphical user interface of the software. It produces a $5 \mathrm{~V}$ DC output at the line print terminal of DB 25 port. This data is isolated by the use of opto-coupler and further processed by the ST12E encoder IC. The ST12E is an 8 bit encoder IC that has been used out in the transmitter section. The RF transmitter section module is responsible for the wireless channel propagation of data.

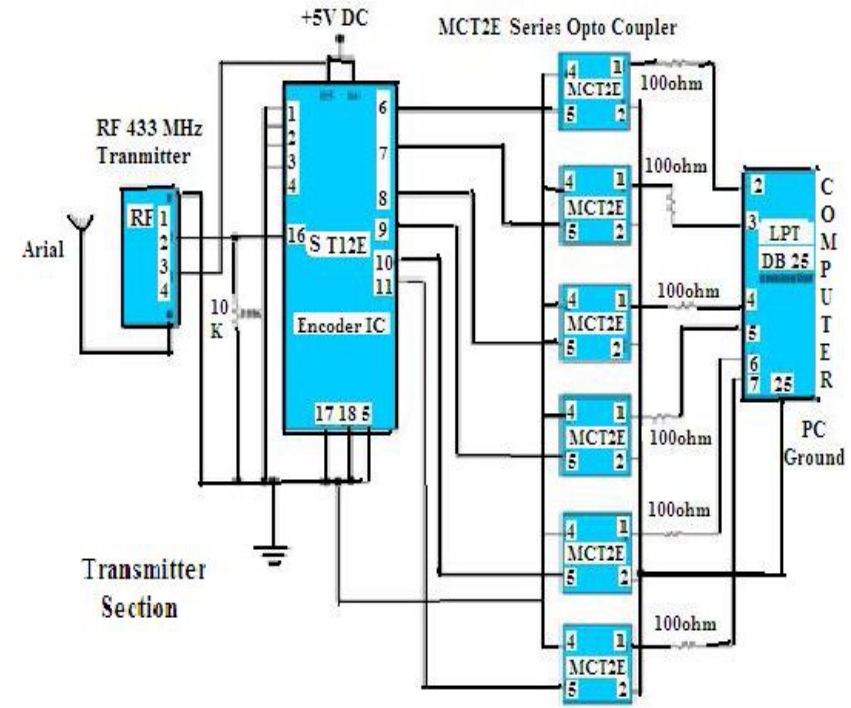

Fig. 6 Implementation of the Interfacing between the RF transmitter and Parallel Port.

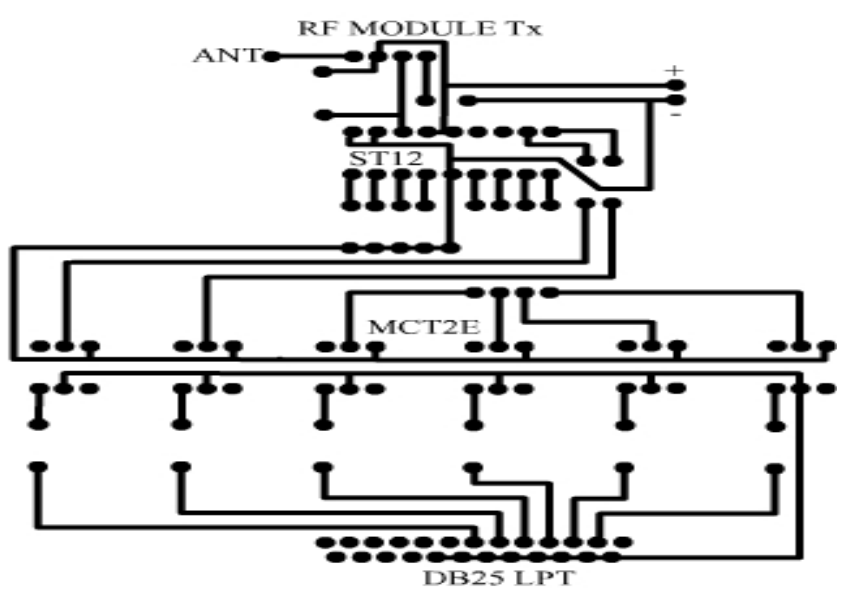

Fig.7 Demonstration of the Developed PCB Lay-out of the Transmitter Assembly.

\section{B. Receiver Section}

At the receiver end, the RF receiver Module receives the encoded data. The RF receiver module is connected with the ST12D IC .The ST12D is an 8 bit decoder IC, which decodes the encoded data. The 8 bit encoded data further processed to operate the three motors, which are connected parallel to the L293 motor driving IC. The pin no. 12 \& 13 of the decoder IC is reserved for the future implementation, which is not discussed in this paper. Now, in order to turn the motor, one of the two motors is turned at a time. Like in padding a boat when the boats need to be turned in one direction, the paddle of other side moved and one side is not disturbed .In this fashion, the boat is turned.

Following the same procedure, for instance, if the drive is to be moved right, the motor controlling the left wheels are tuned forward with the right control left idle. Consequently, the drive is turned right .For the other side, the opposite procedure is followed. Now, the trouble remaining is to prevent the drive from striking any obstruction or falling into hollow cavity on the ground. 


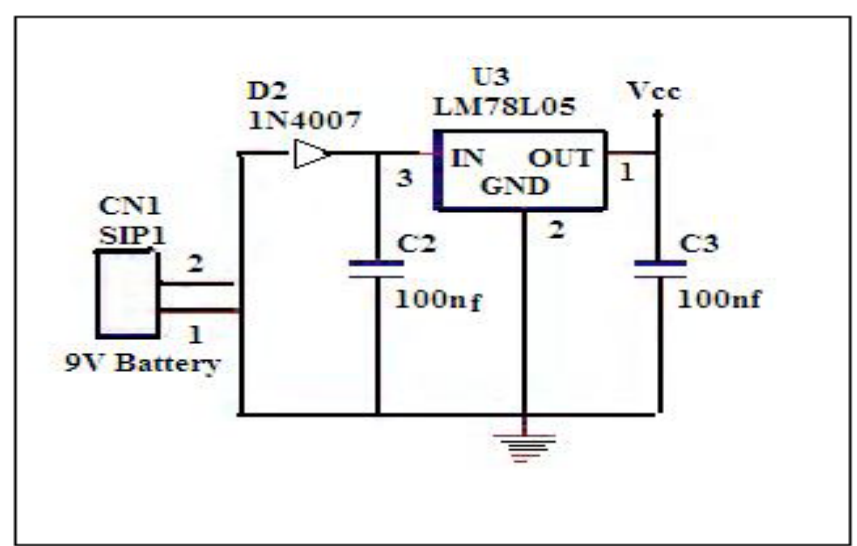

Fig. 8 Representation of the Power Supply Unit at the Receiver End

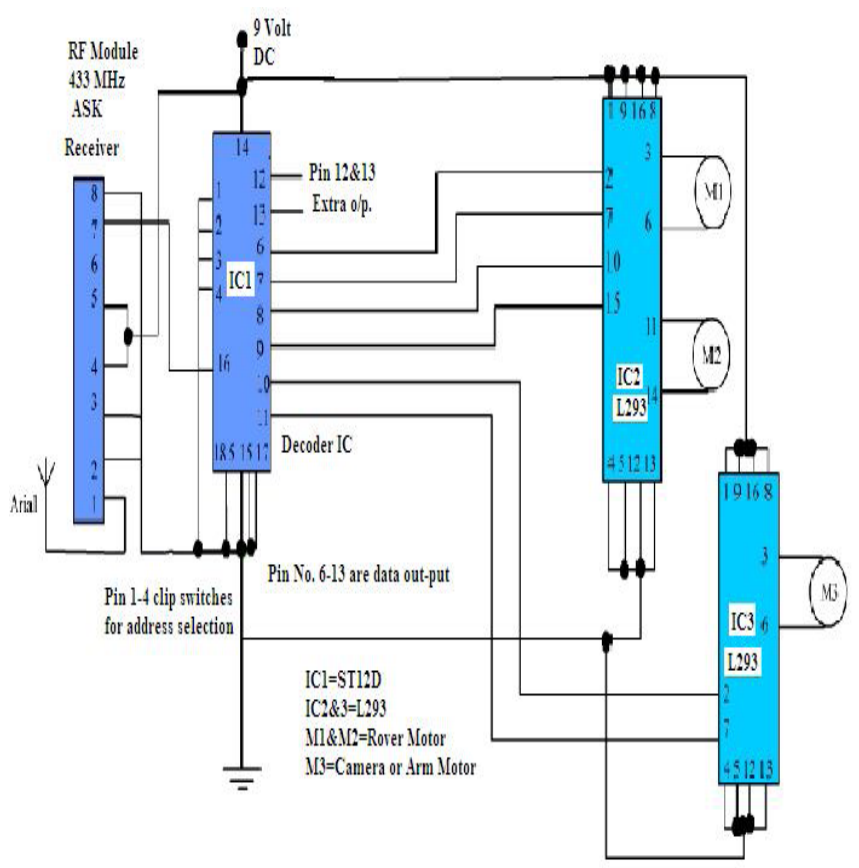

Fig. 9 Implementation of the Interfacing between the RF Receiver and Motors.

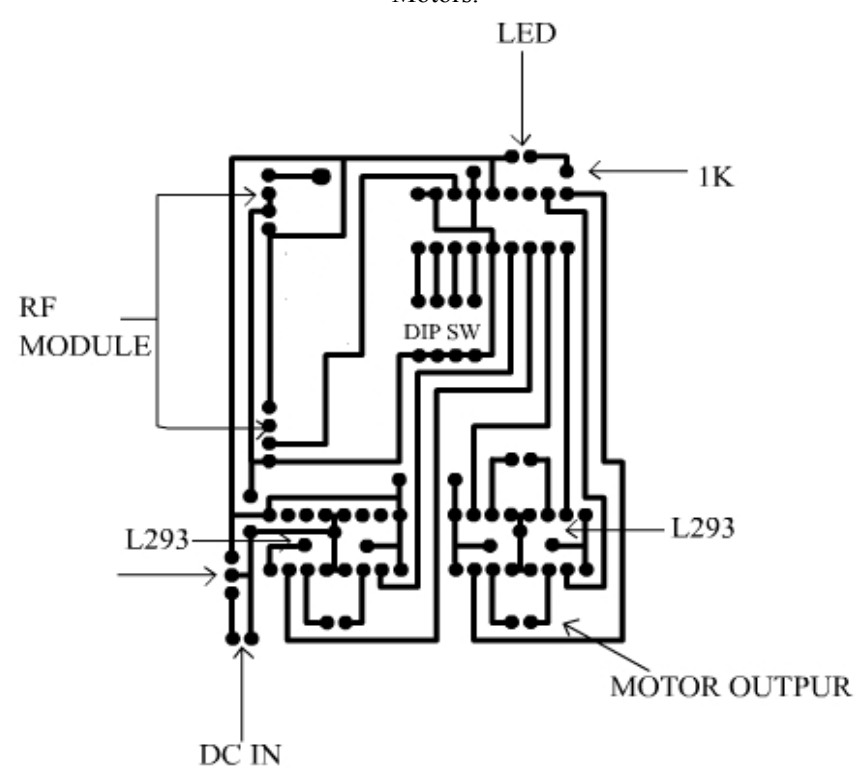

Fig.10 Demonstration of the Developed PCB Lay-out of the Transmitter Assembly.

\section{Implementation of the Wireless CMOS Camera}

The Rotary system units consist out a Stepper motor, which can be able to move in the clockwise and anti clockwise direction by using a gear system. This platform is designed in such a way that it can be able to support the web cam. A wireless CMOS camera is convenient in its highest form. In wireless camera, there are no wires involved and with IP address registry can do everything a normal webcam can do. In area surveillance rover, the wireless CMOS camera is mounted over the rotary platform. The implemented web cam supports $802.11 \mathrm{~g}$ or $802.11 \mathrm{n}$ networking technology. Now for the WPA (Wi-Fi Protected Access) Network by using the encryption algorithm or by simply using the TV tuner card at the PC port we can watch the transmitted image on the mainframe control unit. [3] The resolution power of the wireless camera is $640 * 480$ and the size of camera is $155 \times 85 \times 80 \mathrm{~mm}$. The wireless CMOS camera consist out 3 ports for power, signal and ground .At receiver terminal the power is supplied with the help of $9 \mathrm{~V}$ battery.

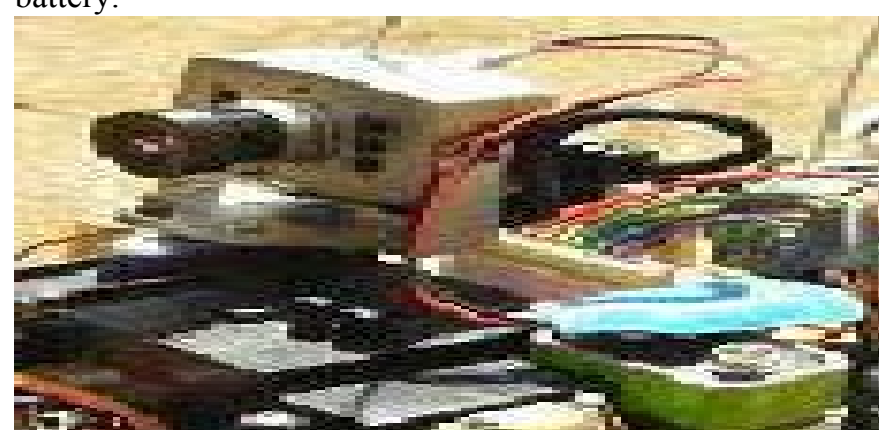

Fig. 11 Interfacing of CMOS camera at the receiver unit of the wireless land rover.

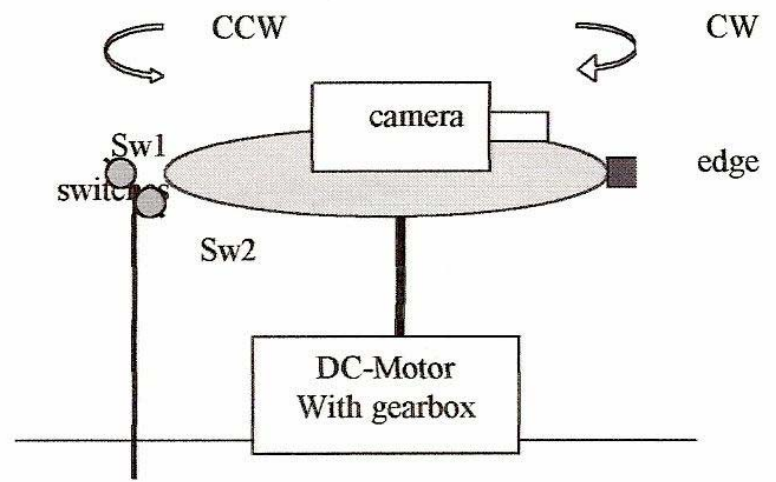

Fig. 12 Demonstration of the Rotary Wheel Platform at the Receiver End of Rover.

The small gear on the Motor drives a larger gear, which drives a larger gear, which drives two larger gears (one on each side), which drive larger gears. The last, largest gears are fixed on shafts that are attached to the front and back wheels, making them move. Note that interlocking gears spin in opposite directions. Also notice that in the sets of interlocking gears between the Motor and the gears on the wheel shafts, the number of "teeth" is increased each time (40-8, 44-8, 64-44, and 64-20), for 128:1 gear ratio overall. This means the Motor must rotate 128 times to rotate the wheels once. The reason for this is that if the Motor were to drive the wheels directly then the Rover would be so fast that it would be impossible to control. Using the gears to reduce the speed also makes the wheels move with much greater force, preventing the Rover from getting stuck in rough 
terrain and allowing it to carry heavy loads uphill. [7]

\section{GUI Implementation}

The GUI is responsible for the generation of control signal. First of all, we have to check the circuit input from the LPT port, which is given by the designed Graphical User Interface [GUI]. For security purpose a Login Password is required to run the software, this password is used here only to provide a legal access to the rover. [4]

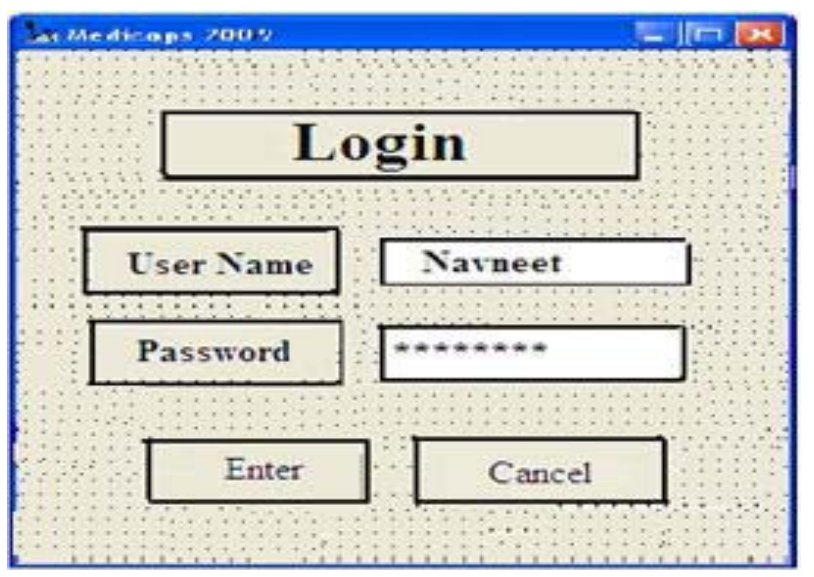

Fig. 13 Demonstration of the Log-In Accession Window of the Rover.

Now by entering the correct user name and password will provide the accession of the Land rover wireless controller window. Then after a successful password entry a rover controller window will appear which is directly linked to the parallel port through the VB programming. Now, every click over the icon generates the relevant control signal and our rover will operate according to our instructions.

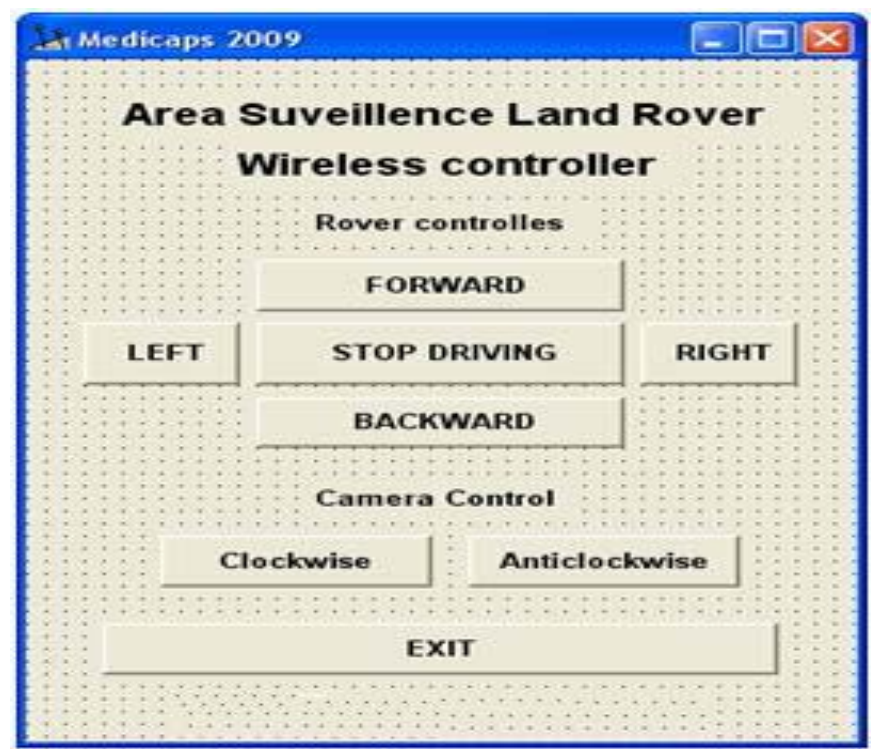

Fig. 14 Demonstration of Area Surveillance Rover wireless controller window.

\section{IMPLEMENTATION PROBLEMS}

The one of the major implementation problem occurs is due to the HT12Codec IC. The HT12 CODEC IC is a 4 bit generally available IC in the market. By using this IC we can easily operate the two motor, but the problem arises when we are shifting toward the 3 motors. After, a very long search the discovered problem is get resolved by implementing the ST12 series Codec IC . This are the 8 bit CODEC IC, which can operate over the radio frequency having the four address and eight bit data.[7] This IC consists out a built in oscillator and can be able to operate over a wide operating voltage range of 2.0 to 5.5 Volts.

\section{RESUltS AND ANALYSIS}

The receiver and the transmitter unit are modeled over the PCB. The graphical user interface unit is designed in the Visual Basic 6.0. . Which is responsible to produce the control signal for the movement of robot in the various directions. The stepper motor is mounted over the rotary system, which consist out a gear system .The mounted camera is capable to move in the clockwise and the anticlockwise direction .Now, by using the CRO, the output of the transmitter and receiver section were measured and drawn below in the following graphs.

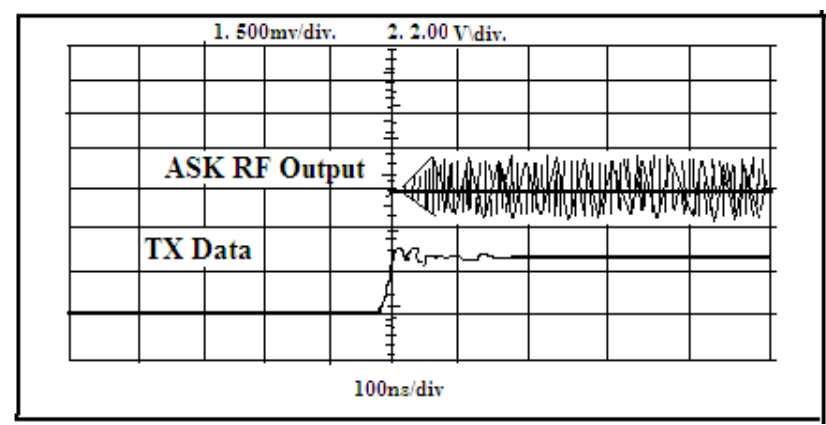

Fig. 15 Performance graph of the Modulation delay In the RF module Transmitter Section.

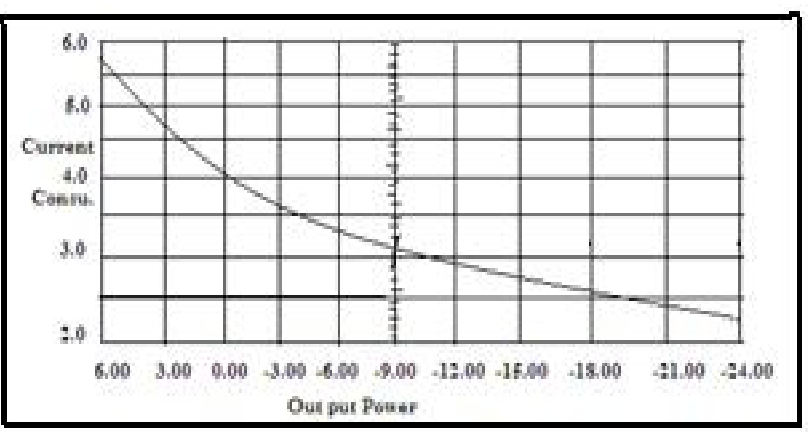

Fig. 16 Performance graph between Current consumption and output power for RF module Transmitter section at $50 \%$ duty cycle.

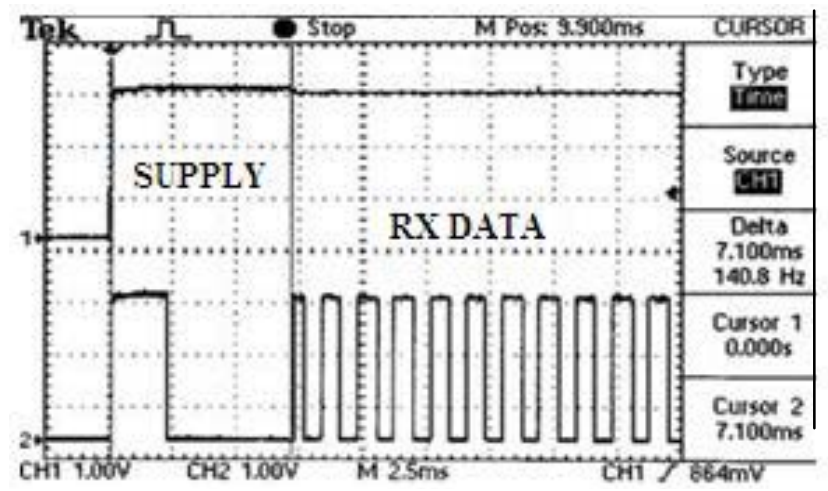

Fig. 17 Graph representing turn on time from Vcc at RX Module Receiver Section. 


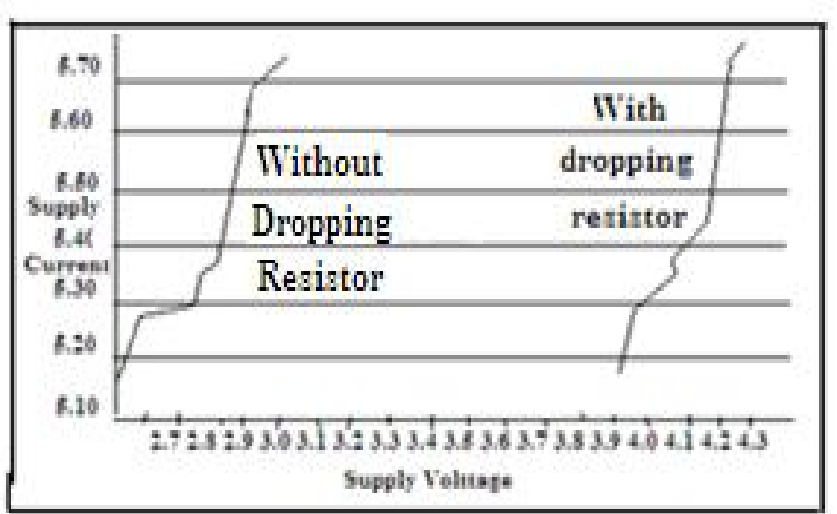

Fig. 18 Performance graph between current Consumption versus Supply for RF Module.

\section{Code Snippet:}

Dim X As Boolean

Private Sub cmdexit_Click()

PortOut \&H378, \& $\overline{\mathrm{HFF}}$

End Sub

Private Sub cmdM1 forward_MouseDown(Button As Integer, Shift As Integer, $\mathrm{X}$ As Single, Y As Single)

PortOut \&H378, \&H1

End Sub

Private Sub cmdM1 forward_MouseUp(Button As Integer, Shift As

Integer, X As Single, Y As Single)

PortOut \&H378, \&H0

End Sub

Private Sub cmdM1 reverse MouseDown(Button As Integer, Shift As

Integer, X As Single, Y As Single)

PortOut \&H378, \&H2

End Sub

Private Sub cmdM1reverse_MouseUp(Button As Integer, Shift As

Integer, X As Single, Y As Single)

PortOut \&H378, \&H0

End Sub

Private Sub cmdcamclock_MouseDown(Button As Integer, Shift As Integer, X As Single, Y As Single)

PortOut \&H378, \&H4

End Sub

Private Sub cmdcamclock_MouseUp(Button As Integer, Shift As

Integer, X As Single, Y As Single)

PortOut \&H378, \&H0

End Sub

Private Sub cmdcamanticlock MouseDown(Button As Integer, Shift

As Integer, X As Single, Y As Single)

PortOut \&H378, \&H8

End Sub

Private Sub cmdcamanticlock_MouseUp(Button As Integer, Shift As Integer, X As Single, Y As Single)

PortOut \&H378, \&H0

End Sub

Private Sub cmdM2forward_MouseDown(Button As Integer, Shift As Integer, X As Single, Y As Single)

PortOut \&H378, \&H10

End Sub

Private Sub cmdM2forward_MouseUp(Button As Integer, Shift As

Integer, X As Single, Y As Single)

PortOut \&H378, \&H0

End Sub

Private Sub cmdM2reverse_MouseDown(Button As Integer, Shift As

Integer, X As Single, Y As Single)

PortOut \&H378, \&H20

End Sub

Private Sub cmdM2reverse_MouseUp(Button As Integer, Shift As Integer, X As Single, Y As Single)

PortOut \&H378, \&H0

End Sub

Private Sub EMoff_MouseUp(Button As Integer, Shift As Integer, X

As Single, Y As Single)

PortOut \&H378, \&H0

End Sub

Private Sub EMon_MouseUp(Button As Integer, Shift As Integer, X
As Single, Y As Single)

PortOut \&H378, \&H40

End Sub

Private Sub Form_Load()

PortOut \&H378, \&H0

End Sub

\section{CONCLUSION AND FUtURE WORK}

For the wireless channel implementation of the Area Surveillance Rover, we have used IEEE 802.11 protocol. The area surveillance rover is designed and tested over the variety of terrain conditions. The wireless camera works effectively in the 100 meter range. We have used out TV tuner for the clear reception of the images. The software has been designed over Visual Basic 6.0, which also works successfully and generates the required control signal. In the future, implementation, we will try to attach a robotic arm over the area surveillance rover. The technology is also moving toward the USB, so we will try to use USB port instead of using parallel port. We have already started our efforts in this direction. Rover can be made water resistive by providing proper shielding/covering to the rover. Any further suggestion, for the improvement of Area Surveillance Rover is cordially invited. Because as we say in the earlier that, "We are looking for endless opportunity".

\section{REFERENCES}

[1] Muhammad Ali Mazidi, and Janice Gillispie Mazidi, "The 8051 Micro -controller and Embedded Systems”, Pearson Education,2000.

[2] Behrouz A. Forouzan, Data Communications and Networking, Prentice Hall, Inc., 2nd Edition 2003.

[3] Holzmann, 'Design and Validation of computer protocols, Prentice Hall Publication,2nd Edition, November 1990.

[4] John Sheriff, "John Sheriff Teaches Visual Basic 6.0”, Prentice Hall of India, 2002.

[5] Coal Mine Fatalities ,'Fatal gram and Investigation Report 1996 to 2005", Mine Safety and Health Administration, United States Department of Labor .

[6] http:// www.fairchildsemi.com/pf/1N/1N4007.html

[7] http:// www.electricomconsulting.co.uk/overview.html

[8] http://www.fbo.gov/spg/ODA/DARPA/CMO/BAA06\%2D36 /Atta chments.htm 1. The DARPA Grand Challenge web site [www . darpa. $\mathrm{Mil} /$ grandchallenge]

[9] William Stallings, "Wireless Communication and Networks", Pearson Education, 2002. 